International Journal of Network Security \& Its Applications (IJNSA), Vol.4, No.3, May 2012

\title{
Multi Stage Filter Using Enhanced Adaboost for Network Intrusion Detection
}

\author{
P.Natesan ${ }^{1}$, P.Balasubramanie ${ }^{2}$ \\ Department of Computer Science and Engineering, \\ Kongu Engineering College, Perundurai, Erode 638 052, Tamilnadu, India \\ ${ }^{1}$ natesanp@gmail.com, ${ }^{2}$ pbalu_20032001@yahoo.co.in
}

\begin{abstract}
Based on the analysis and distribution of network attacks in KDDCup99 dataset and real time traffic, this paper proposes a design of multi stage filter which is an efficient and effective approach in dealing with various categories of attacks in networks. The first stage of the filter is designed using Enhanced Adaboost with Decision tree algorithm to detect the frequent attacks occurs in the network and the second stage of the filter is designed using enhanced Adaboost with Naïve Byes algorithm to detect the moderate attacks occurs in the network. The final stage of the filter is used to detect the infrequent attack which is designed using the enhanced Adaboost algorithm with Nä̈ve Bayes as a base learner. Performance of this design is tested with the KDDCup99 dataset and is shown to have high detection rate with low false alarm rates.
\end{abstract}

Keywords: Enhanced Adaboost, multi stage filter, decision tree, Naive Bayes classification, detection rate, false alarm rate.

\section{Introduction}

With the development of the Internet, web applications are becoming increasingly popular and it plays an important role in human life. Consequently, the various Internet resources are becoming the major targets of many attacks. Due to the growing number of users, networking components and the applications in the Internet, it is mandatory that development of new techniques that can secure and protect the Internet resources against the various attacks. These issues have given rise to a research on Network Intrusion Detection systems.

The intrusion detection is a vital part of network security. Intrusion detection is the detection of user behaviors in the network that deviates from the organizations network security policy. The goals of network intrusion detection are to identify, categorize and possibly respond to malicious or suspicious activities $[1,2]$. There are basically two types of intrusion detection systems namely anomaly detection and misuse detection. Anomaly detection system first learns normal system activities and then alerts all system events that deviate from the learned model and the misuse detection uses the signature of attacks to detect intrusions by modeling attacks.

\subsection{Related work}

The field of network intrusion detection and network security has been around since late 1990s. Since then, a number of frameworks and methodologies have been proposed and many tools have been built to detect network intrusion. Various methodologies such as rule based DOI : $10.5121 /$ ijnsa.2012.4308 
International Journal of Network Security \& Its Applications (IJNSA), Vol.4, No.3, May 2012

algorithm, classification, clustering, genetic algorithms, support vector machines, hybrid classification and others have been used to detect network intrusions. In this section, we briefly discuss few of these methodologies and frameworks.

Weiming Hu et. al., [3] have proposed an Adaboost based algorithm for network intrusion detection. The AdaBoost algorithm is one of the most popular machine learning algorithms and it corrects the misclassifications made by weak classifiers. The authors in [3] used decision stump as a weak classifier. The decision rules are provided for both categorical and continuous features and some provision was made for handling the overfitting.

N.B.Amor et. al, [4] discuss the use of Decision tree and Naïve Bayes classifiers for network intrusion detection. The decision trees select the best features for each decision node during the construction of the tree based on some well defined criteria. Decision trees generally have very high speed of operation and high attack detection accuracy. The Naïve Bayes classifiers make strict independence assumption between the features in an observation resulting in lower attack detection accuracy when the features are correlated, which is often the case for intrusion detection.

Dewan Md. Farid et. al., [5] constructed an adaptive intrusion detection system by combining Naïve Bayes and Decision tree algorithms. The various issues of intrusion detection system are addressed in this model and its performance is better in terms of attack detection rate and false positive rate with limited computational resources.

Natesan et. al, [6] proposed the use of multiple base learners with Adaboost algorithm. The Decision tree algorithm, Naïve Bayes and Bayes Net are used as base learners and it is also combined in three different ways with Adaboost and its performance is better in terms of Attack detection rate and false alarm rate. Xiang et al [7] proposed multiple-level Hybrid Classifier (MLHC), which involved both the supervised classification stages and unsupervised Bayesian clustering to detect intrusions. There are four classification stages in hybrid classifier which uses Bayesian clustering and decision tree technologies. Gupta et al [8] proposed a Layered approach using Conditional Random Fields (CRFs), where it is considered that the attack categories as layers and different features were selected for each layer.

Chin khor et al [9] proposed a cascaded classifier approach in which they divided the training set in to non-rare attack categories and rare attack categories and trained the classifiers using these two training datasets. The methodologies used in the cascaded classifier approach were Bayes net and C4.5 decision tree. Natesan et. al [10] proposed a two stage filter in which the Adaboost with Decision tree methodology is used in the first stage and the Adaboost with Bayes Net methodology is used in the second stage of the filter. In our work, we define three stage filters, first stage is to filter the DoS attacks which occur frequently in the network and the second stage is to filter Probe attacks which occur moderately in the network. The third stage of the filter is used to filter the R2L and $\mathrm{U} 2 \mathrm{R}$ attacks which occur rarely in the network. This approach improves the detection rate of Probe attacks and also the detection of novel attacks improved. 
International Journal of Network Security \& Its Applications (IJNSA), Vol.4, No.3, May 2012

\subsection{Dataset Analysis}

Under the sponsorship of Defense Advanced Research Projects Agency (DARPA) and Air Force Research Laboratory (AFRL), MIT Lincoln laboratory has collected and distributed the datasets for the evaluation of researches in computer network intrusion detection systems. The KDDCup99 dataset is subsets of the DARPA benchmark dataset [11].

KDDCup99 training dataset is about four giga bytes of compressed binary TCP dump data from seven weeks of network traffic, processed into about five million connections record each with about 100 bytes. The two weeks of test data have around two million connection records. Each KDDCup'99 training connection record contains 41 features and is labeled as either normal or an attack, with exactly one specific attack type [11].

There are about 494,020 records in KDDCup'99 training set and 311,029 records in the KDDCup'99 test set. The various attack types in the datasets are grouped into attack categories in order to combine similar attack types into a single category which could improve the detection rate. The training set consists of 24 attack types and the test set contains 38 attack types in which 14 attack types are novel attacks. All the attacks in the dataset fall into four major categories, namely, Denial of Service (Dos), Probing (Probe), Remote to Local (R2L) and User to Root $(U 2 R)$. Table.1 shows the attacks in the KDDCup'99 training set and the additional attacks present in the KDDCup'99 test set.

Table 1: Attacks present in the KDDCup'99 Datasets

\begin{tabular}{|l|l|l|}
\hline $\begin{array}{l}\text { Attack } \\
\text { Category }\end{array}$ & Attacks in KDDCup'99 Training set & $\begin{array}{l}\text { Additional attacks in KDDCup'99 } \\
\text { Test set }\end{array}$ \\
\hline Dos & $\begin{array}{l}\text { back, neptune, smurf, teardrop, land, } \\
\text { pod. }\end{array}$ & apache2, mailbomb, processtable. \\
\hline Probe & satan, portsweep, ipsweep, nmap. & mscan, saint. \\
\hline R2L & $\begin{array}{l}\text { warezmaster, warezclient, ftpwrite, } \\
\text { guesspassword, imap, multihop, phf, } \\
\text { spy }\end{array}$ & $\begin{array}{l}\text { sendmail, named, snmpgetattack, } \\
\text { snmpguess, xlock, xsnoop, worm. }\end{array}$ \\
\hline U2R & $\begin{array}{l}\text { rootkit, bufferoverflow, loadmodule, } \\
\text { perl. }\end{array}$ & httptunnel, ps, sqlattack, xterm \\
\hline
\end{tabular}

Table. 2 and Table. 3 shows the number of records for each attack category in the training and testing datasets respectively.

Table 2: Number of samples in the KDDCup'99 Training set and distribution of attacks

\begin{tabular}{|l|r|r|}
\hline $\begin{array}{l}\text { Attack } \\
\text { Category }\end{array}$ & $\begin{array}{l}\text { Number of } \\
\text { samples }\end{array}$ & $\begin{array}{l}\text { Distribution of } \\
\text { Attacks in \% }\end{array}$ \\
\hline Normal & 97,277 & 19.6909 \\
\hline Dos & 391,458 & 79.2393 \\
\hline Probe & 4,107 & 0.8313 \\
\hline R2L & 1,126 & 0.2279 \\
\hline U2R & 52 & 0.0105 \\
\hline Total & 494,020 & 100 \\
\hline
\end{tabular}


International Journal of Network Security \& Its Applications (IJNSA), Vol.4, No.3, May 2012

Table 3: Number of samples in the KDDCup'99 Test set and distribution of attacks

\begin{tabular}{|l|r|r|}
\hline $\begin{array}{l}\text { Attack } \\
\text { Category }\end{array}$ & $\begin{array}{l}\text { Number } \\
\text { of samples }\end{array}$ & $\begin{array}{l}\text { Distribution of } \\
\text { Attacks in \% }\end{array}$ \\
\hline Normal & 60,593 & 19.4814 \\
\hline Dos & 229,853 & 73.9008 \\
\hline Probe & 4,166 & 1.3394 \\
\hline R2L & 16,189 & 5.2049 \\
\hline U2R & 228 & 0.0733 \\
\hline Total & 311,029 & 100 \\
\hline
\end{tabular}

The percentage of distribution of attacks is not uniform in the training set and the test set. Also, the probability of distribution of attacks in the test set is different from training set. For example, there are about only $0.2 \%$ of R2L attacks in the training set but it is about $5.2 \%$ in the test set. This is one of the challenging tasks in the classification of attacks.

\section{Methodology in building IDS}

This section will give a detailed description about the Rough set theory which is used to extract the relevant features for detecting the specific category of attacks from the generic 41 features present in the KDDCup99 dataset. With the selected features, the enhanced Adaboost algorithm can achieve the low false alarm rate with high attack detection rate. This section also discuss about the enhanced Adaboost algorithm.

\subsection{Rough set theory}

Z.Pawlak introduced the Rough set theory in the early 1980s, is an extension of set theory for study of the intelligent systems characterized by insufficient and incomplete information [12]. Recently, rough set theory has attracted a lot of attention and has been applied in the areas of patterns extraction, text classification, machine learning, information retrieval and etc. [13$15]$.

We present an overview of rough set theory and the notations used in this paper. In rough set theory, an information system, which is also called a decision table, is defined as $S=\{\mathrm{U}, \mathrm{A}$, $V, f\}$, where $U=\left\{U_{1}, U_{2}, \ldots, U_{m}\right\}$ is a non-empty, finite set of objects called universe. $A=\left\{a_{1}\right.$, $\left.a_{2}, \ldots a_{n}\right\}$ is a non-empty, finite set of attributes. Here, $m$ is the no.of objects and $n$ is the number of attributions. It includes two non-intersect subsets: one is condition attributes subset $\mathrm{C}$ and another is decision attributes subset $D$, namely $A=C U D, C \cap D=\Phi . V=U V_{a}(a \epsilon A)$ is a set of values of attributes in $A, V_{a}$ is called the domain of $a$.

$\mathrm{F}$ : $\mathrm{U} x \mathrm{~A} \rightarrow \mathrm{Va}$ is an information function, for any

a $\epsilon \mathrm{A}, \mathrm{x} \epsilon \mathrm{U}, \mathrm{f}(\mathrm{x}, \mathrm{a}) \boldsymbol{\epsilon} \mathrm{Va}$

The reduction of attributes means to find the minimum condition attribution subset whose classification quality is identical to the original condition attribution set.

Suppose $U$ is the universe $R$ is a group of equivalence relation, $r R$, if $U \mid \operatorname{IND}(R)=U$ I $\operatorname{IND}(\mathrm{R}-\mathrm{r})$, then we say $r$ can be deducted from $\mathrm{R}$. If every attribution in $\mathrm{P}=\mathrm{R}-\{\mathrm{r}\}$ cannot be deducted, then we say $\mathrm{P}$ is a reduction of $\mathrm{R}$. 
International Journal of Network Security \& Its Applications (IJNSA), Vol.4, No.3, May 2012

\subsection{Enhanced Adaboost Algorithm}

AdaBoost is a fast machine learning algorithm, can be used in conjunction with many other learning algorithms to improve their performance. It calls a weak classifier repeatedly in a series of rounds. In each round of operation the classification error is calculated for various categories of attacks. The reweight is calculated and assigned to the instances. The pseudo code of our Enhanced Adaboost algorithm is given.

Input: $D$, a set of $d$ class-labeled, $\mathrm{n}$ training network connections

$\mathrm{k}$, the number of rounds, apply a classification learning scheme.

Output: An IDS model.

Steps:

(1) Split the training dataset in to three datasets $\mathrm{D}_{1}, \mathrm{D}_{2}$ and $\mathrm{D}_{3}$

(2) Extract the relevant features by applying Rough set

(3) Initialize the weight of each network connection in $D_{x}$ to $1 / d$; $\mathrm{x}$ is the number

of

Splits,

(4) For $\mathrm{x}=1$ to 3 do

(5) $\quad$ For $i=1$ to $k$ do

(6) Sample $D_{i}$ with replacement according to the network connection weights to obtain $D_{s}$;

(7) Use training set $D_{s}$ to derive a model, $M_{i}$;

(8) Calculate error $\left(M_{i}\right)$, the error rate of the model $M_{i}$

(9) if $\operatorname{error}\left(M_{i}\right)>0.5$ then

(10) Reinitialize the weights to $1 / d$

End if

Go back to step 5 and try again;

For each network connection in $D_{s}$ that was correctly classified

Do

Update the weight of the network connection by

Error $\left(\mathrm{M}_{\mathrm{i}}\right)=\left(1\right.$-Error $\left.\left(\mathrm{M}_{\mathrm{i}}\right)\right)$;

Normalize the weight of each network connection End for

The Enhanced Adaboost algorithm uses C4.5 Decision tree and Naïve Bayes classification algorithms as its base learner.

\section{Experiments}

The various stages involved in our experiments are: splitting the training dataset, filter design and feature selection, training the filter and testing the model using KDDCup99 test set.

\subsection{Splitting the training dataset}

The KDDCup99 training dataset is split in to three training datasets. The first one consisted of records of Normal, DoS attack categories and the second one consisted of Normal, Probe attack categories. The third split consisted of records of Normal, R2L and U2R attack categories. 
International Journal of Network Security \& Its Applications (IJNSA), Vol.4, No.3, May 2012

\subsection{Filter design and Feature selection}

The large number of features in the KDDCup99 dataset increases the computational and space cost, besides the redundant characteristics of the attributes make the attack detection accuracy dropped. Hence, to reduce the cost involved in computation and storage, rough set based feature selection was applied to select features that were relevant to detect the frequent attacks, moderate attacks and infrequent attacks in the KDDCup99 Training set. We have proposed a multi stage filter which consists of three stages as shown in Figure.1, the first stage is used to filter the DoS attacks and the second stage is for filtering Probe attacks in the network. The final stage is used to filter the R2L and U2R attacks. The proposed method is called a multi stage filter with Adaboost.

Figure .1: Block Diagram of Multi Stage filter

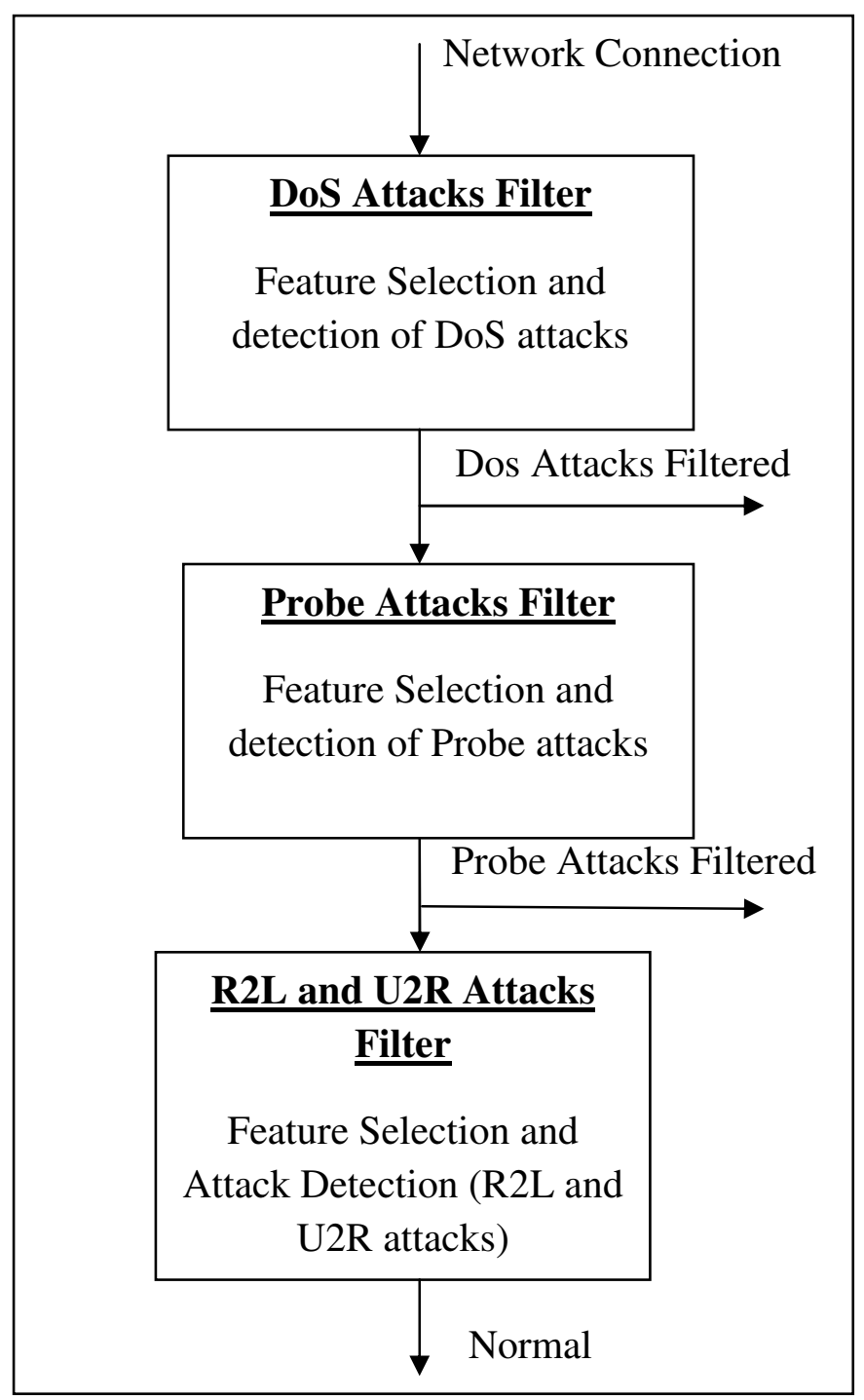


International Journal of Network Security \& Its Applications (IJNSA), Vol.4, No.3, May 2012

\subsubsection{DoS attacks detection stage}

This stage of the filter is aimed to detect Dos attacks which occur frequently in the network. In Dos attacks the attacker makes some computing or memory resources too busy or too full to handle legitimate requests or denies legitimate users access to a machine. The selected features to detect the DoS attacks are shown in Table.4.

Table. 4: Features selected for DoS attacks detection stage

\begin{tabular}{|c|l|l|}
\hline $\begin{array}{c}\text { Feature } \\
\text { Number }\end{array}$ & Feature Name & Description \\
\hline 1 & Duration & Length of the connection in seconds \\
\hline 2 & protocol_type & Type of the protocol used. For eg. TCP \\
\hline 3 & Service & $\begin{array}{l}\text { Network service on the destination like HTTP, } \\
\text { Telnet. }\end{array}$ \\
\hline 4 & Flag & $\begin{array}{l}\text { Normal or error status of the network } \\
\text { connection. }\end{array}$ \\
\hline 5 & src_bytes & $\begin{array}{l}\text { Number of data bytes from source to } \\
\text { destination. }\end{array}$ \\
\hline 6 & dst_bytes & $\begin{array}{l}\text { Number of data bytes from destination to } \\
\text { source. }\end{array}$ \\
\hline 23 & Count & $\begin{array}{l}\text { Number of connections to the same host as the } \\
\text { current connection in the past two seconds. }\end{array}$ \\
\hline 25 & serror_rate & $\begin{array}{l}\text { Percentage of connections that have "SYN" } \\
\text { errors. }\end{array}$ \\
\hline 27 & rerror_rate & $\begin{array}{l}\text { Percentage of connections that have "REJ" } \\
\text { errors. }\end{array}$ \\
\hline 30 & diff_srv_rate & $\begin{array}{l}\text { Percentage of connections that have same } \\
\text { services. }\end{array}$ \\
\hline 32 & dst_host_count & Count for destination host. \\
\hline 33 & dst_host_srv_count & srv_count for destination host \\
\hline 34 & dst_host_same_srv_rate & same_srv_rate for destination host \\
\hline 35 & dst_host_diff_srv_rate & diff_srv_rate for destination host. \\
\hline 39 & dst_host_srv_serror_rate & srv_serror_rate for destination host. \\
\hline
\end{tabular}

\subsubsection{Probe attacks detection stage}

This stage of the filter is aimed to detect Probe attacks which happen moderately in the network. Probe attacks scan a network to gather information or to find known vulnerabilities. An intruder with a map of machines and services that are available on a network can use the information to look for exploits. The selected features to detect the Probe attacks are shown in Table.5.

Table. 5: Features selected for Probe attacks detection stage

\begin{tabular}{|c|l|l|}
\hline $\begin{array}{c}\text { Feature } \\
\text { Number }\end{array}$ & Feature Name & Description \\
\hline 1 & Duration & Length of the connection in seconds \\
\hline 2 & protocol_type & Type of the protocol used. For eg. TCP \\
\hline 3 & Service & Network service on the destination like HTTP, \\
\hline
\end{tabular}


International Journal of Network Security \& Its Applications (IJNSA), Vol.4, No.3, May 2012

\begin{tabular}{|c|l|l|}
\hline & & Telnet. \\
\hline 5 & src_bytes & $\begin{array}{l}\text { Number of data bytes from source to } \\
\text { destination. }\end{array}$ \\
\hline 6 & dst_bytes & $\begin{array}{l}\text { Number of data bytes from destination to } \\
\text { source. }\end{array}$ \\
\hline 19 & num_access_files & Number of operations on access control files \\
\hline 20 & number_outbound_cmds & $\begin{array}{l}\text { Number of outbound commands in an ftp } \\
\text { session }\end{array}$ \\
\hline 23 & Count & $\begin{array}{l}\text { Number of connections to the same host as the } \\
\text { current connection in the past two seconds. }\end{array}$ \\
\hline 25 & serror_rate & $\begin{array}{l}\text { Percentage of connections that have "SYN" } \\
\text { errors. }\end{array}$ \\
\hline 27 & rerror_rate & $\begin{array}{l}\text { Percentage of connections that have "REJ" } \\
\text { errors. }\end{array}$ \\
\hline 30 & diff_srv_rate & $\begin{array}{l}\text { Percentage of connections that have same } \\
\text { services. }\end{array}$ \\
\hline 32 & dst_host_count & Count for destination host. \\
\hline
\end{tabular}

\subsubsection{R2L and U2R attacks detection stage}

This stage of the filter is aimed to detect R2L and U2R attacks which happen rarely in the network. Remote to User (R2L) is an attack that a remote user gains access of a local user/account by sending packets to a machine over a network communication. User to Root (U2R) is an attack that an intruder begins with the access of a normal user account and then becomes a root-user by exploiting various vulnerabilities of the system. The selected features to detect the R2L and U2R attacks are shown in Table. 6.

Table. 6: Features selected for R2L and U2R attacks detection stage

\begin{tabular}{|c|l|l|}
\hline $\begin{array}{c}\text { Feature } \\
\text { Number }\end{array}$ & Feature Name & Description \\
\hline 1 & Duration & Length of the connection in seconds \\
\hline 2 & protocol_type & Type of the protocol used. For eg. TCP \\
\hline 3 & Service & Network service on the destination like HTTP, Telnet. \\
\hline 4 & Flag & Normal or error status of the network connection. \\
\hline 5 & src_bytes & Number of data bytes from source to destination \\
\hline 6 & dst_bytes & Number of data bytes from destination to source. \\
\hline 10 & Hot & Number of "hot" indicators. \\
\hline 11 & num_failed_logins & Number of failed login attempts. \\
\hline 12 & logged-in & 1 if successfully logged in; 0 otherwise. \\
\hline 13 & num_compromised & Number of compromised conditions. \\
\hline 14 & root_shell & 1 if root shell is obtained; 0 otherwise. \\
\hline 16 & num_root & Number of "root" access. \\
\hline 17 & num_file_creations & Number of file creation operations. \\
\hline 18 & num_shells & Number of shell prompts. \\
\hline 19 & num_access_files & Number of operations on access control files. \\
\hline 21 & is_host_login & 1 if the login belongs to "hot" list; 0 otherwise. \\
\hline
\end{tabular}


International Journal of Network Security \& Its Applications (IJNSA), Vol.4, No.3, May 2012

\begin{tabular}{|l|l|l|}
\hline 22 & is_guest_login & 1 if the login is a guest login; 0 otherwise. \\
\hline 23 & Count & $\begin{array}{l}\text { Number of connections to the same host as the current } \\
\text { connection in the past two seconds }\end{array}$ \\
\hline 25 & serror_rate & Percentage of connections that have "SYN" errors. \\
\hline 27 & rerror_rate & Percentage of connections that have "REJ" errors. \\
\hline 30 & diff_srv_rate & Percentage of connections that have same services. \\
\hline 32 & dst_host_count & Count for destination host. \\
\hline 35 & dst_host_diff_srv_rate & diff_srv_rate for destination host. \\
\hline 39 & dst_host_srv_serror_rate & srv_serror_rate for destination host. \\
\hline
\end{tabular}

\subsection{Training and Testing the Model}

The multi stage filter is constructed using the selected features in Tables. 4,5 \& 6 . There are 15 features selected for the first stage of the filter which is used for detecting the DoS attacks which occurs frequently in the network and 12 features selected for the construction of the second stage of the filter which is used to filter out the Probe attacks which occurs moderately in the network. The third stage of the filter is constructed to filter out the R2L and $\mathrm{U} 2 \mathrm{R}$ attacks which occur rarely in the network. There are 24 features selected for this stage to filter out the R2L and U2R attacks. The learning ability of any classification algorithm is dependent on the characteristics of the connections in the training data set.

The Enhanced Adaboost with Decision tree algorithm is used in constructing the first stage of the filter and it is trained with the training set which consists of Normal and DoS attack categories. The second stage of the filter is constructed by using the Enhanced Adaboost with Naïve Bayes classification algorithm as its base learner. The training dataset which consists of Normal and Probe attack categories is used in training the second stage of the filter. The third stage of the filter is also constructed by using the Enhanced Adaboost with Naïve Bayes classification algorithm as its base learner. The training dataset which consists of Normal, R2L and U2R attack categories is used in training the third stage of the filter. The trained model is tested using the KDDCup99 test set.

\section{Experiments and Results}

For our experiments we use the benchmark KDDCup99 dataset, in which each record represents a separate connection and hence each connection between the two IP addresses is considered to be independent of any other connection. We use the Weka tool to implement the Enhanced Adaboost algorithm and to perform classification with Decision Trees and naïve Bayesian classification.

\subsection{Performance Measures}

In machine learning and data mining algorithms, many different metrics are used to evaluate the classification models. We employed two performance measures: Attack detection rate and false alarm rate. These two measures are calculated by using a confusion matrix. 
International Journal of Network Security \& Its Applications (IJNSA), Vol.4, No.3, May 2012

Confusion matrix is a two dimensional matrix representation of the classification results. The upper left cell in the matrix denotes the number of connections classified as Normal while they actually were Normal (i.e. TP), and the lower right cell in the matrix denotes the number of connections classified as Attack while they actually were Attack (i.e. TN). The other two cells (lower left cell and the upper right cell) denote the number of connections misclassified. Specifically, the lower left cell in the matrix denoting the number of connections classified as Normal while they actually were Attack(i.e.FN), and the upper right cell denoting the number of connections classified as Attack while they actually were Normal (i.e. FP).

\begin{tabular}{|l|c|c|}
\hline & $\begin{array}{c}\text { Classified as } \\
\text { Normal }\end{array}$ & $\begin{array}{c}\text { Classified as } \\
\text { Attack }\end{array}$ \\
\hline Normal & TP & FP \\
\hline Attack & FN & TN \\
\hline
\end{tabular}

Attack Detection Rate (ADR): It is the ratio between total numbers of attacks detected by the system to the total number of attacks present in the dataset.

$$
\text { Attack Detection Rate }=\frac{\text { Total detected attacks }}{\text { Total attacks }} * 100
$$

False Alarm Rate (FAR): It is the ratio between total numbers of misclassified instances to the total number of normal instances.

$$
\text { False Alarm Rate }=\frac{\text { Total misclassified instances }}{\text { Total normal instances }} * 100
$$

\subsection{Detecting the DoS attacks}

We conducted two sets of experiments. In the first experiment, we considered all the 41 features of the dataset and examined the detection rate of the DoS attacks which occurs frequently in the network. Initially the Decision Tree is constructed and then to improve its classification accuracy the enhanced Adaboost algorithm is used. We perform the same experiment with the 15 features selected as in Table. 4 by using Enhanced Adaboost with Decision Tree as its base learner. The experimental results are shown in Table 7.

Table 7: The attack detection rate of first stage of the filter

\begin{tabular}{|c|c|c|c|}
\hline $\begin{array}{c}\text { No. of } \\
\text { features }\end{array}$ & $\begin{array}{c}\text { \% of detection } \\
\text { rate }\end{array}$ & $\begin{array}{c}\text { Training } \\
\text { Time(sec) }\end{array}$ & $\begin{array}{c}\text { Test } \\
\text { Time(sec) }\end{array}$ \\
\hline 41 & 97.8 & 9.7 & 0.43 \\
\hline 15 & 98.9 & 6.2 & 0.26 \\
\hline
\end{tabular}

The system takes only $6.2 \mathrm{sec}$ for its training when 15 features considered. Also it takes only $0.26 \mathrm{sec}$ for the testing of the incoming network connection. 
International Journal of Network Security \& Its Applications (IJNSA), Vol.4, No.3, May 2012

\subsection{Detecting the Probe attacks}

We conducted two sets of experiments. In the first experiment, we considered all the 41 features of the dataset and examined the detection rate of Probe attacks which happened moderately in the network. Initially the Naïve Bayes classification algorithm is chosen and then to improve its classification accuracy the enhanced Adaboost algorithm is used. We perform the same experiment with the 12 features selected as in Table. 5 by using Enhanced Adaboost with Naïve Bayes as its base learner. The experimental results are shown in Table 8.

Table 8: The attack detection rate of second stage of the filter

\begin{tabular}{|c|c|c|c|}
\hline $\begin{array}{c}\text { No. of } \\
\text { features }\end{array}$ & $\begin{array}{c}\text { \% of detection } \\
\text { rate }\end{array}$ & $\begin{array}{c}\text { Training } \\
\text { Time(sec) }\end{array}$ & $\begin{array}{c}\text { Test } \\
\text { Time(sec) }\end{array}$ \\
\hline 41 & 92.3 & 6.9 & 0.36 \\
\hline 12 & 93.8 & 2.7 & 0.17 \\
\hline
\end{tabular}

The system takes only $2.7 \mathrm{sec}$ for its training when 12 features considered. Also it takes only $0.17 \mathrm{sec}$ for the testing of the incoming network connection.

\subsection{Detecting the $R 2 L$ and $U 2 R$ attacks}

To detect the infrequent attacks, initially we conducted an experiment by considering all the 41 features. The Naïve Bayes algorithm is used as a base learner with the enhanced Adaboost algorithm. We perform the same experiment with 24 features which are relevant to detect infrequent attacks and the results are shown in Table 9. The training time and the testing time decreases marginally with the selected 24 features and there is a slight increase in the detection rate of attacks.

Table 9: The attack detection rate of third stage of the filter

\begin{tabular}{|c|l|c|c|c|}
\hline $\begin{array}{c}\text { No. of } \\
\text { features }\end{array}$ & $\begin{array}{c}\text { Attacks } \\
\text { category }\end{array}$ & $\begin{array}{c}\text { \% of detection } \\
\text { rate }\end{array}$ & $\begin{array}{c}\text { Training } \\
\text { Time(sec) }\end{array}$ & $\begin{array}{c}\text { Test } \\
\text { Time(sec) }\end{array}$ \\
\hline \multirow{3}{*}{41} & Normal & 98.8 & \multirow{2}{*}{14.7} & 0.83 \\
\cline { 2 - 3 } & R2L & 44.7 & & \\
\cline { 2 - 3 } & U2R & 82.6 & \multirow{2}{*}{11.2} & \multirow{2}{*}{0.62} \\
\hline \multirow{2}{*}{24} & Normal & 99.3 & & \\
\cline { 2 - 3 } & R2L & 49.8 & \\
\cline { 2 - 3 } & U2R & 87.6 & & \\
\hline
\end{tabular}

The false alarm rate of this multi stage filter is found to be $1.97 \%$ which is less as compared with the two stage filter [10]. 


\subsection{System performance on Novel attacks}

The KDDCup99 test dataset contains some specific type of new attacks that did not present in the KDDCup99 training dataset and this makes the classification task is more challenging. There are about 24 types of attacks in the training set and 14 types of additional novel attacks present in the test dataset as shown in the Table. 1. The network domain experts and intrusion detection system experts suggested that most of these attacks are slight variants of known attacks which are present in training set and the "patterns" of known attacks can be sufficient to detect the novel attacks. We conducted an experiment to test the performance of our system on novel attacks with the selected features and the results are shown in Table.10.

Table 10: Detection rate on Novel attacks

\begin{tabular}{|l|l|r|r|}
\hline $\begin{array}{l}\text { Attacks } \\
\text { Category }\end{array}$ & Attacks Name & $\begin{array}{c}\text { Number of attack } \\
\text { connections in } \\
\text { KDDCup99 test set }\end{array}$ & $\begin{array}{c}\text { Number of } \\
\text { attacks detected }\end{array}$ \\
\hline Dos & apache2 & 794 & 575 \\
& mailbomb & 5,000 & 4,569 \\
process table & 759 & 613 \\
\hline Probe & mscan & 1,053 & 989 \\
& saint & 736 & 736 \\
\hline U2R & httptunnel & 158 & 121 \\
& ps & 16 & 11 \\
& sqlattack & 2 & 1 \\
& xterm & 13 & 10 \\
\hline R2L & sendmail & 17 & 12 \\
& named & 17 & 5 \\
& snmpgetattack & 7,741 & 3,865 \\
& snmpguess & 2,406 & 1,986 \\
& xlock & 9 & 2 \\
& xsnoop & 4 & 1 \\
& worm & 2 & 0 \\
\hline & Total & 18,729 & $13,496(72.1 \%)$ \\
\hline
\end{tabular}

The performance of our multi stage filter with Adaboost on novel attacks is remarkably high (an increase of 32.5\%) as compared with the work in [19].

\subsection{Comparisons of detection rate with different algorithms}

The detection rate of our algorithm is compared with existing work as shown in Table. 11, which is tested on the benchmark KDDCup'99 dataset. The performance of our proposed two stage filter was comparatively better than existing work in detecting the DoS, R2L and U2R attacks (DoS - 98.8\%, R2L - 49.8\% and U2R - 87.6\%). Hence, it should be considered for the building of IDS. 
International Journal of Network Security \& Its Applications (IJNSA), Vol.4, No.3, May 2012

Table 11: Comparison with other algorithms

\begin{tabular}{|l|c|c|c|c|c|}
\hline \multirow{2}{*}{ Name of the method } & \multicolumn{5}{|c|}{ \% of Detection rate } \\
\cline { 2 - 6 } & Normal & DoS & Probe & R2L & U2R \\
\hline $\begin{array}{l}\text { Cascaded classifier } \\
\text { using J48-BN [9] }\end{array}$ & 97.4 & 97.8 & 73.3 & 48.2 & 87.3 \\
\hline Multi layered hybrid classifier [7] & 96.8 & 98.6 & 93.4 & 46.9 & 71.4 \\
\hline KDD'99 Winner[21] & $\mathbf{9 9 . 5}$ & 97.1 & 83.3 & 8.4 & 13.2 \\
\hline Layered approach using CRFs[8] & N/A & 97.4 & $\mathbf{9 8 . 6 2}$ & 29.6 & 86.3 \\
\hline $\begin{array}{l}\text { Two stage filter using enhanced } \\
\text { Adaboost [10] }\end{array}$ & 99.2 & 98.7 & 92.4 & 49.5 & 87.5 \\
\hline $\begin{array}{l}\text { Proposed Multi stage filter using } \\
\text { enhanced Adaboost }\end{array}$ & 98.8 & $\mathbf{9 8 . 9}$ & 93.8 & $\mathbf{4 9 . 8}$ & $\mathbf{8 7 . 6}$ \\
\hline
\end{tabular}

\section{Conclusion and Future work}

We have proposed the Rough set theory for extracting relevant features and the Enhanced Adaboost algorithm for detecting the network intrusion. The experiment is conducted with all 41 features and with the selected features. The attacks detection rate is increased considerably with the selected features and the computational cost falls drastically. The various issues of intrusion detection system such as attack detection rate, false alarm rate and computational time for building robust, scalable and efficient system are addressed. It is important to have very quick attack detection with higher detection rate. The experiment result shows that the Rough set theory with enhanced Adaboost algorithm has quick attack detection with a high detection rate.

The areas for future research include the considering use of multi core processors in which each stage of the filter is configured to individual core of the processor which will improve the utilization of the processor and also the attraction detection time.

\section{References}

1. Xuan Dau Hoang, Jiankun Hu, and Peter Bertok, "A program-based anomaly intrusion detection scheme using multiple detection engines and fuzzy inference," Journal of Network and Computer Applications, Vol. 32, pp. 1219-1228, 2009.

2. P. Garcia-Teodoro, J. Diaz-Verdejo, G. Macia-Fernandez, and E.Vazquez, "Anomaly-based network intrusion detection: Techniques, systems and challenges," Computers \& Security, Vol. 28, pp. 18-28, 2009.

3. Weiming $\mathrm{Hu}$, Wei Hu and Steve Maybank, "AdaBoost-Based Algorithm for Network Intrusion Detection," IEEE Transactions on Systems, Man and Cybernetics, Vol. 38, pp. 577-583, April2008. 
International Journal of Network Security \& Its Applications (IJNSA), Vol.4, No.3, May 2012

4. N. B. Amor, S. Benferhat, and Z. Elouedi, "Naïve Bayes vs. decision trees in intruison detection systems," In Proc. of the 2004 ACM Symposium on Applied Computing, New York, pp. 420424, 2004.

5. Dewan Md. Farid, Nouria Harbi and Mohammad Zahidur Rahman, "Combining naive bayes and decision tree for adaptive intrusion detection", International Journal of Network Security \& its Applications, Vol. 2, No. 2, pp. 12 - 25, 2010.

6. Natesan P, Balasubramanie P and Gowrison G, "Adaboost with Single and Compound weak classifier in Network Intrusion Detection", In Proceedings of International conference on Advanced computing, Networking \& Security", Vol. 1, pp 282-290, Dec-2011.

7. Xiang C, Yong PC, Meng, "Design of multiple-level hybrid classifier for intrusion detection system using Bayesian clustering and decision trees". Pattern Recognition Letters 29(7):918924, 2008.

8. Gupta KK, Nath B (2010) Layered approach using conditional random fields for intrusion detection. IEEE Trans Dependable Secure Computing 7(1):35-49, 2010.

9. Kok-Chin Khor, Choo-Yee Ting and Somnuk Phon-Amnuaisuk A, "cascaded classifier approach for improving detection rates on rare attack categories in network intrusion detection", Journal of Applied Intelligence, DOI 10.1007/s10489-010-0263-y.

10. Natesan P, Balasubramanie $P$ and Gowrison G, "Design of two stage filter using enhanced Adaboost for improving attack detection rates in network intrusion detection", Journal of Computer science, Information technology and Security, Vol.2, No.2, pp. 349-357.

11. KDDCup99 Dataset, http://kdd.ics.uci.edu/databases/kddcup99/ kddcup99.html. 1999.

12. Z.Pawlak, "Rough sets", International Journal of Computer and Information Sciences, Vol.11, No. 5, pp. 341-356, 1982.

13. Lei Shi, Li Zhang, Xinming $\mathrm{Ma}$ and Xiaohong Hu, "Rough set Based Personalized Recommendation in Mobile Commerce. 2009 International conference on Active Media Technology", Lecture Notes in Computer Science, 370-375, 2009.

14. Sabhnani, M. R., \& Serpen, G. Application of machine learning algorithms to KDD intrusion detection dataset with in misuse detection context. In Proceedings of the international conference on machine learning: Models, technologies, and applications. pp. 209-215, 2003.

15. Xuren, W., Famei, H., \& Rongsheng, X. Modeling intrusion detection system by discovering association rule in rough set theory framework. In Proceedings of the international conference on computational intelligence for modeling control and automation, and international conference on intelligent agents. Web Technologies and Internet Commerce (CIMCAIAWTIC'06), 2006.

16. J.W. Han and M.Kamber, Data Mining: Concepts and Techniques, $2^{\text {nd }}$ edition. Morgan Kaufmann, pp. 310-318, 2006.

17. N.Friedman, D.Geiger and M.Goldsmidt, "Bayesian Network Classifiers," Machine Learning, Vol.29, pp 131-163, Nov 1997.

18. Yoav Freund, Robert E.Schapire. "A Decision theoretic generalization of on-line learning and an application to boosting," Journal of Computer and System Sciences. Vol. 55, 119-139, 1997.

19. P.N. Tan, Introduction to Data Mining. Reading, MA:Addison-Wesley, 2006.

20. Shi-Jinn Horng, Ming-Yang Su, Yuan-Hsin Chen, Tzong-Wann kao, Rong-Jian Chen, Jui-Lin Lai, Citra Dwi Perkasa, "A novel intrusion detection system based on hierarchical clustering and 
International Journal of Network Security \& Its Applications (IJNSA), Vol.4, No.3, May 2012

support vector machines", Expert Systems with Applications, Vol.38, pp.306-313, 2010, DOI:10.1016/j.eswa.2010.06.066.

21. Pfahringer, B. Winning the KDD99 classification cup: Bagged boosting SIGKDD Explorations, $1(2), 65-66,2000$.

\section{AUTHORS PROFILE}

P.Natesan has completed his M.E., (Computer Science \& Engineering) in Anna University, Tamilnadu, India in 2005 . Now he is doing research in the field of Network Security. Currently, he is working as Associate Professor in the Department of Computer Science \& Engineering, Kongu Engineering College, Tamil Nadu, India. He has completed 15 years of teaching service. He has published 5 articles in International / National journals.

Dr.P.Balasubramanie has completed his Ph.D., degree in Theoretical Computer Science in 1996 in Anna University. He was awarded Junior Research Fellow in the year 1990 by CSIR. Currently he is a Professor in the department of Computer Science \& Engineering in Kongu Engineering College, Tamilnadu, India. He has completed 17 years of teaching service. He has published more than 100 articles in International /National Journals. He has authored 8 books with reputed publishers. He has guided $14 \mathrm{Ph} . \mathrm{D}$., scholars and guiding 10 research scholars. He is serving as a Editorial/Advisory board member for many Journals.
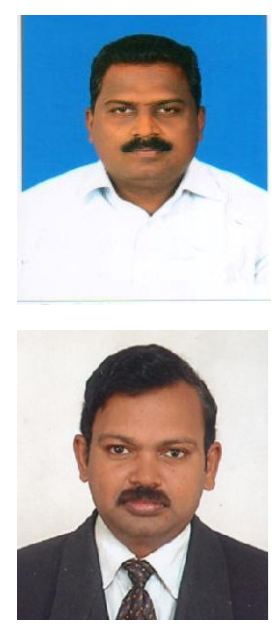